\title{
L5 vertebral osteomyelitis treated with L5 corpectomy and anterior instrumentation: case report
}

\author{
Authors Kelli L Crabtree ${ }^{1}$, Angela Spurgeon ${ }^{2}$, Harel Arzi ${ }^{3}$, Bryan Beaver ${ }^{4}$, Paul M Arnold ${ }^{1}$ \\ Institution $\quad{ }^{1}$ Department of Neurosurgery University of Kansas Medical Center, Kansas City, KS, USA \\ 2 Department of Neurosurgery, University of Missouri Medical School, Columbia, MO, USA \\ ${ }^{3}$ Department of Orthopedics, The Spine Deformity Unit, The Chaim Sheba Medical Center, \\ Tel Hashomer, Israel \\ ${ }^{4}$ School of Medicine, University of Kansas Medical Center, Kansas City, KS, USA
}

\begin{abstract}
Study design: A case report.

Objective: Pyogenic osteomyelitis is the most common form of vertebral infection and typically resolves following conservative treatment with antibiotics administered long term and immobilization. In cases of spinal instability, severe neurological deficit or disease refractory to medical management, neurosurgical intervention is warranted. Historically, these patients have undergone radical vertebral debridement and grafting with or without posterior instrumentation. We report the case of a 46-year-old female intravenous drug user presenting with L5 pyogenic osteomyelitis with L5 vertebral compression and cortex retropulsion following L2-L4 laminectomy for epidural abscess 8 weeks prior.
\end{abstract}

Methods: The patient underwent an anterior approach single-stage L5 corpectomy, L4/5 and L5/S1 discectomies, expandable titanium-cage insertion and anterior plating from L4 to the sacrum.

Results: The patient recovered without any complications. The infection was successfully eradicated and her fusion remains solid 18 months postoperatively.

Conclusions: To our knowledge, this is the first case of L5 vertebral osteomyelitis treated with a singlestage corpectomy and anterior instrumentation. 


\section{INTRODUCTION}

Although pyogenic osteomyelitis is the most common form of vertebral body infection, pyogenic vertebral osteomyelitis only accounts for $2-4 \%$ of all bone infections [1]. While antibiotics given long term are considered the mainstay of treatment, surgical intervention is necessary in cases of neurological compromise, unstable mechanical deformity, intractable pain, or progressive disease refractory to maximal medical management. Recent studies have shown that surgical therapy is indicated in up to $57 \%$ of patients with pyogenic vertebral osteomyelitis, a rate much higher than previously believed $[2,3,6,7]$. In cases of vertebral osteomyelitis requiring surgery, L5 is affected only $4-9 \%$ of the time [4-7].

The surgical technique most commonly reported for the treatment of L5 osteomyelitis is single-stage anterior debridement and stabilization with a titanium mesh cage and posterior instrumentation [5-7]. We describe the case of a 46-year-old woman with a history of L2-L4 laminectomy for epidural abscess presenting with L5 vertebral osteomyelitis secondary to a new pathogen. The patient underwent a single-stage L 5 corpectomy, L4/5 and L5/S1 discectomies, insertion of an expandable titanium cage, and application of an anterior plate from L4 to S1 via an anterior approach. To our knowledge, this is the first case of L5 vertebral osteomyelitis treated surgically with an anterior approach, single-stage corpectomy with anterior instrumentation.

\section{CASE REPORT}

A 46-year-old woman was transferred from an outside hospital secondary to liver failure and altered mental status following acetaminophen overdose. Her medical history was significant for intravenous drug abuse, hepatitis C, bipolar disorder, schizophrenia, and the recent onset of sciatica. Initial neurological examination was limited by mental status. She was able to follow commands and move fingers and toes bilaterally but unable to generate any more proximal movements. She was hypotonic with decreased rectal tone. Sensation appeared intact. The patient had extensive edema and subcutaneous abscesses in all four extremities. Further workup revealed an L2 to L4 epidural abscess, which was likely responsible for her neurological compromise. Subsequently the patient underwent an urgent decompressive L2-L4 laminectomy. Intraoperative cultures were positive for methicillin-sensitive Staphylococcus aureus. Intravenous antibiotic therapy was started. Postoperatively her mental status and strength improved significantly. Once she remained afebrile with adequate pain control, she was discharged to a long-term care facility for inpatient rehabilitation and continued intravenous antibiotic therapy.

Approximately 2 months later, the patient was transferred again to our institution with severe lower back pain in addition to combined vancomycin-resistant enterococcus and Citrobacter freundii bacteremia. On examination, the patient was neurologically intact with brisk reflexes noted bilaterally in the lower extremities. She was able to stand but refused to walk secondary to pain. The lumbar incision wound was well healed; mild fluctuance was present bilaterally in the paraspinous region. Lumbar magnetic resonance imaging identified destructive changes from osteomyelitis at L4-L5 resulting in secondary compression of the L5 vertebral body with retropulsion of the posterior cortex. Extensive epidural soft tissue was also noted from L4 to S2, causing both central canal and right neuroforaminal stenosis from L4 to S1. Marrow edema was seen at L4 and L5 (Fig 1). Computed tomography of the lumbar spine showed L4/5 discitis with extensive bony destruction of adjacent L4/5 end plates and collapse of L5 (Fig 2). A biopsy of the L5 vertebral body revealed chronic osteomyelitis.

Given the progression of the patient's infection despite antibiotic treatment, collapse of the L 5 vertebral body and extensive scar formation, the decision was made to perform an L5 corpectomy and fusion using an expandable titanium cage and titanium plate with an anterior-only approach. Anterior dissection revealed an inflammatory mass around the anterior aspect of the vertebra with 
Fig 1 T1-weighted sagittal postcontrast magnetic resonance imaging shows L5 osteomyelitis with disc space and vertebral body enhancement, as well as collapse of L5. Postoperative laminectomy is also evident.

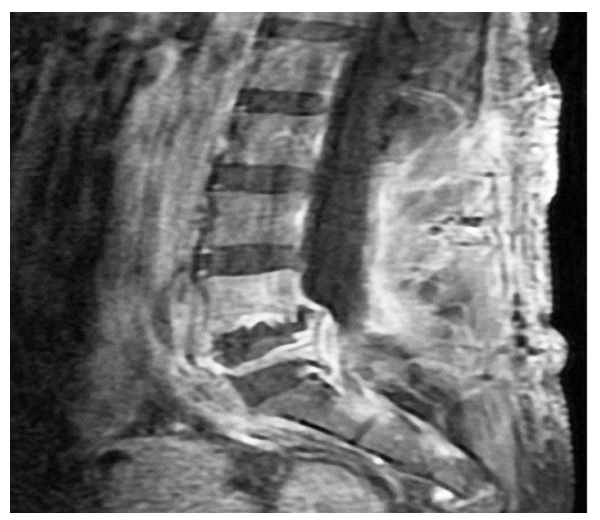

Fig 2 Sagittal reformatted computed tomography shows collapse of L5, as well as previous laminectomy.

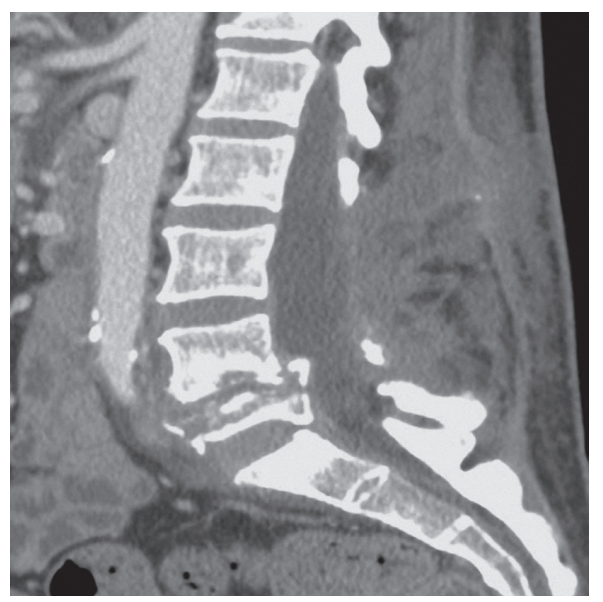

Fig 3 Postoperative computed tomography shows reconstruction of L5 with titanium cage and restoration of lordosis.

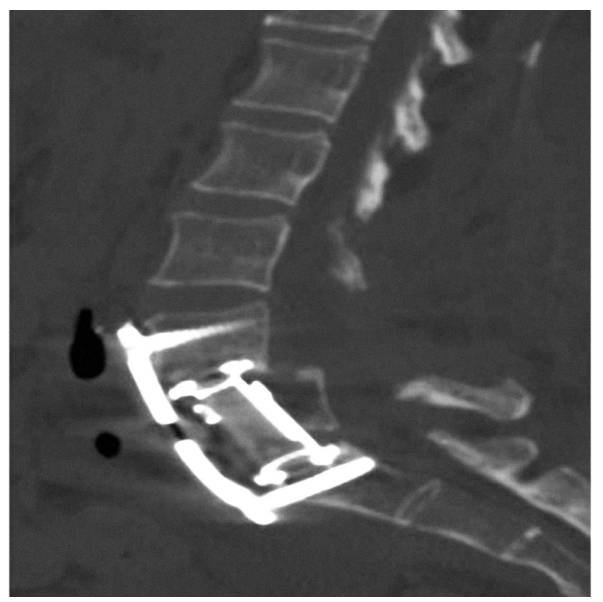

the vessels tightly adherent to it. After careful dissection, the left common iliac artery and vein were freed. Discectomy of L4/5 and L5/S1 was performed in addition to L5 corpectomy with nearly complete removal of the L5 vertebral body. An expandable titanium cage filled with demineralized bone matrix and allograft was used to reconstruct the $\mathrm{L} 5$ vertebral body. A titanium plate was then inserted under the iliac vein from L4 to Sl and secured with screws at S1 and L4. Postoperative imaging showed that all instrumentation was in place. Intraoperative cultures did not isolate any organisms. Overall the patient tolerated the procedure well without any complications.

After surgery it was determined the infection was largely controlled, and the patient was discharged and given oral antimicrobial therapy. She was followed-up 18 months after discharge and has done fine without any recurrence of infection. Follow-up lumbar computed tomography shows solid fusion from L4 to S1 with good lumbar lordosis and no evidence of lumbar stenosis (Fig 3).

\section{DISCUSSION}

Pyogenic osteomyelitis is the most common form of vertebral body infection. Conservative treatment with antibiotics administered long term and immobilization is usually sufficient but cases of spinal instability, severe neurological deficit or disease refractory to medical management warrant neurosurgical intervention [1-3]. Recent studies have demonstrated that radical anterior debridement and stabilization with a titanium cage and posterior instrumentation is an effective surgical treatment for vertebral osteomyelitis of the lumbar spine [5-8]. However, L5 involvement requiring corpectomy is exceedingly rare. Lu et al [7] reported the highest incidence of spinal osteomyelitis affecting L5; seven of 36 patients had L5 involvement and underwent L 5 corpectomy with posterior instrumentation. Korovessis et al [5] reported one anterior L5 corpectomy with posterior instrumentation for further stabilization of 24 cases reviewed. In a series by Kuklo et al [6], two of 22 osteomyelitis cases were treated with L5 corpectomy with insertion of a mesh cage and posterior instrumentation. Risks associated with the combined anterior and posterior approach include increased morbidity related to prolonged anesthesia and operative time, as well as additional blood loss and tissue damage. Although the literature has demonstrated that posterior instrumentation is an effective method of stabilization after L5 corpectomy, a posterior approach was unfavorable in this case due to massive scar formation and the partial deficiency of posterior elements after extensive debridement during the first operation. 
The challenge of preserving lumbar lordosis is also an important consideration when attempting to provide stability after L5 corpectomy. Approximately two-thirds of lumbar lordosis is created by the discs at L4/5 and L5/S1, both of which were removed in this case due to infection and subsequent vertebral body compression [9]. In the case presented, an expandable titanium cage was selected to aid in the reconstruction of the anterior column.

Studies have recently examined the role of expandable titanium cages in the treatment of spinal osteomyelitis. Liljenzvist et al [10] reported promising results in the treatment of 20 patients with vertebral osteomyelitis treated with single-stage posterior instrumentation and fusion with anterior debridement and decompression. The anterior column was reconstructed using expandable titanium cages filled with morsellised autogenous bone graft. In the 18 cases involving the lumbar spine, lordosis was significantly corrected at follow-up. There was no evidence of cage dislocation, migration, or subsidence noted in any case, and all infections were eliminated. In a review of 36 cases of vertebral osteomyelitis treated with corpectomy and anterior column reconstruction with an expandable titanium cage, an anterior stand-alone corpectomy and reconstruction was performed in only five cases [7]. Most patients underwent combined anterior and posterior approaches, including the seven patients with some degree of L5 involvement. In an effort to preserve lordosis, the authors used an expandable titanium cage typically reserved for the thoracic spine and placed the cage so that the curve corresponds with lumbar lordosis rather than thoracic kyphosis. The study concluded that expandable cages with allograft have a low rate of recurrent infection and are both safe and effective in patients with pyogenic vertebral osteomyelitis requiring surgery [7].

An anterior approach is preferred in the surgical treatment of pyogenic vertebral osteomyelitis as it allows for adequate debridement and reconstruction of the affected tissue, which is almost always anterior to the spinal canal $[11,12]$. Following debridement and reconstruction, the use of posterior instrumentation is a logical choice as the infectious process in this field is believed to be controlled. The success of anterior insertion of titanium cages eases the concern of placing metallic constructs into an infected area, making anterior plating a viable option. A prospective series by Dia et al [4] has demonstrated that anterior plating was effective treatment for 22 patients with pyogenic vertebral osteomyelitis in both the thoracic and lumbar spine. In these cases, corpectomies and discectomies were performed, and then followed with bone strut autografts or titanium mesh cages for anterior fusion. Each patient was then treated with anterior spinal plating using titanium Z-plate instrumentation extending one level above and below the affected vertebrae. Solid fusion was achieved and the infection was successfully eradicated in all cases [4]. However, none of these cases had any L5 involvement requiring corpectomy. Other case reports have also demonstrated success with anterior instrumentation in the setting of osteomyelitis; however, these case series were small and less than five patients per series had lesions in the thoracolumbar spine [12-16]. Although single-stage anterior corpectomy with anterior instrumentation has been successful in vertebral osteomyelitis, concerns regarding the placement of hardware into an infected field remain [17-19]. Considering the most recent data supporting anterior instrumentation and the limited surgical options for this patient, the decision was made to perform the anterior debridement with L5 corpectomy, L4/5 and L5/S1 discectomies, and place an anterior titanium plate extending from the body of L4 to S1, rather than the combined anterior and posterior approaches.

\section{CONCLUSION}

Single-stage treatment of L 5 osteomyelitis consisting of anterior L5 corpectomy, anterior column reconstruction with an expandable titanium cage, and anterior titanium plate has been a safe and effective treatment for our patient. At 18 months follow-up, she remains solidly fused without any recurrent infection. By using this anterior-only technique, the risks associated with prolonged operative time, greater blood loss, and additional tissue damage seen in an anteroposterior surgery could be decreased. This indicates the possibility of an anterior-only approach as a safe and effective alternative to the traditional method of anterior debridement with insertion of a titanium mesh cage plus posterior instrumentation in patients with vertebral osteomyelitis requiring L5 corpectomy. 


\section{REFERENCES}

1. Kulkowski J (1936) Pyogenic osteomyelitis of the spine: an analysis and discussion of 102 cases. J Bone Joint Surg Am; 18:343-364.

2. Hadjipavlou AG, Mader JT, Necessary JT, et al (2000) Hematogenous pyogenic spinal infections and their surgical management. Spine; 25:1668-1679.

3. McHenry MC, Easley KA, Locker GA (2002) Vertebral osteomyelitis: long-term outcome for 253 patients from seven Cleveland-area hospitals. Clin Infect Dis; 34:1342-1350.

4. Dia L-Y, Chen W-H, Jiang L-S (2008) Anterior instrumentation for the treatment of pyogenic vertebral osteomyelitis of thoracic and lumbar spine. Eur Spine J; 17:1027-1034.

5. Korovessis $\mathbf{P}$, Repantis $\mathbf{T}$, Iliopoulos $\mathbf{P}$, et al (2008) Beneficial influence of titanium mesh cage on infection healing and spinal reconstruction in hematogenous septic spondylitis: a retrospective analysis of surgical outcome of twenty-five consecutive cases and review of literature. Spine; 33:E759-E767.

6. Kuklo TR, Potter BK, Bell RS, et al (2006) Single-stage treatment of pyogenic spinal infection with titanium mesh cages. J Spinal Disord Tech; 19:376-382.

7. Lu DC, Wang V, Chou D (2009) The use of allograft or autograft and expandable titanium cages for the treatment of vertebral osteomyelitis. Neurosurgery; 64:122-130.

8. Ruf M, Stoitze D, Merk HR, et al (2007) Treatment of vertebral osteomyelitis by radical debridement stabilization using titanium mesh cages. Spine; 32:E275-E280.

9. Canale ST, Beaty JH (2008) Campbell's Operative Orthopaedics. 11th ed. Vol 2. Philadelphia: Mosby-Elsevier, 2295.

10. Liljenzvist $U$, Lerner $T$, Bullman $\mathbf{V}$, et al (2003) Titanium cages in the surgical treatment of severe vertebral osteomyelitis. Eur Spine J; 12:606-612.

11. Ozuna RM, Delamarter RB (1996) Pyogenic vertebral osteomyelitis and postsurgical disc space infection. Orthop Clin North Am; 27:87-94.

12. Rezai AR, Woo HH, Errico TJ, et al (1999) Contemporary management of spinal osteomyelitis. Neurosurgery; 44:1018-1026.

13. Faraj AA, Webb JK (2000) Spinal instrumentation for primary pyogenic infection: report of thirty-one patients. Acta Orthop Belg; 66:242247.

14. Kostuik JP (1983) Anterior spinal cord decompression for lesions of the thoracic and lumbar spine, techniques, new methods of internal fixation results. Spine; 8:512-531.

15. Nather A, David V, Hee HT, et al (2005) Pyogenic vertebral osteomyelitis: a review of fourteen cases. J Orthop Surg; 13:240-244.
16. Woertgen C, Rothoerl RD, Englert C, et al (2006) Pyogenic spinal infections and outcome according to the 36 -item short form health survey. J Neurosurg Spine; 4:441-446.

17. Hee H, Majd M, Holt R, et al (2002) Better treatment of vertebral osteomyelitis using posterior stabilization and titanium mesh cages. J Spinal Disord; 15:149-156.

18. Lee MC, Wang MY, Fessler RG, et al (2004) Instrumentation in patients with spinal infection. Neurosurg Focus; 17:E7.

19. Safran O, Rand N, Kaplan L, et al (1998) Sequential or simultaneous, same-day anterior decompression and posterior stabilization in the management of vertebral osteomyelitis of the lumbar spine. Spine; 23(17):1885-1890. 


\section{COMMENTARY}

Author James M Schuster

Institution Department of Neurosurgery, University of Pennsylvania, Philadelphia, PA, USA

The author describes surgical treatment of $L 5$ osteomyelitis after failure of laminectomy and antibiotic therapy. Because of the amount of vertebral body destruction, surgery would have to reconstruct the incompetent anterior column with stabilization. Generally an anterior approach for further decompression and reconstruction with either allograft, expandable, or fixed length cage would be used. It is sometimes difficult to seat a graft or cage between L4 and S1 because of the obliquity of the sacrum even with cages with variable angle end plates. Stabilization options include a simultaneous anterior stabilization as described by the authors versus a subsequent posterior stabilization. Anterior stabilization, while allowing a one-stage procedure, may require mobilization of the iliac vessels, which can be difficult in an infected surgical bed. However, in this case the previous posterior approach would also make posterior stabilization more difficult. One other option would be a one-stage posterior corpectomy, expandable cage reconstruction, and pedicle screw stabilization [1]. However, because of infection and a previous posterior approach, this would be least advisable.

I would have performed a similar anterior approach with an expandable cage and allograft [2], but because of difficulty mobilizing the iliac vessels, I would have performed a subsequent posterior/pedicle screw stabilization.

- Initial therapy for osteomyelitis is bacterial-specific antibiotic therapy unless there is neurological compromise, progressive deformity, or failure of medical management.

- Surgical treatment must decompress neural elements, debride infected and devitalized tissues, reconstruct structural defects, and stabilize unstable segments.

- The surgical approach must consider the levels of involvement and the surgeons experience and familiarity with the various surgical options, and possible access to an experienced surgeon.

1. Hunt T, Shen FH, Arlet V (2006) Expandable cage placement via a posterolateral approach in lumbar spine reconstructions: technical note. J Neurosurg Spine; 5(3):271-274.

2. Schuster JM, Avellino A, Mann F, et al (2000) The use of structural allografts in spinal osteomyelitis: a review of 47 cases. J Neurosurg Spine; 93(1): 8-14.

\section{EDITORIAL PERSPECTIVE}

The case presented by Crabtree and colleagues and the commentary by Schuster perfectly epitomize a quandary of modern spine surgery. Tried and true surgical therapies - as commented by Schuster-in form of an anterior corpectomy of the collapsed and infected $L 5$ vertebral body followed by anterior column reconstruction with a structural graft (eg, an allograft, an autogenous tricortical graft, or some form of structural material cage with bone-graft filling) and followed by a posterior decompression and stable segmental fixation is outflanked by a more aggressive but elegant single-stage anterior decompression, reconstruction, and stabilization with a new device designed for a different indication (in this case designed for the treatment of degenerative lumbosacral disc disease).

Clearly the latter technique is appealing by virtue of obviating the need for a supplemental posterior procedure. However, this perceived advantage comes at the price of unknown and likely less favorable biomechanical stability at the notoriously complex lumbosacral junction. The fixation device used, an appropriately named 'Anterior Tension Band Plate,' was designed for less destabilizing one-or two-level anterior discectomies and fusion in presence of degenerative diseases at the lumbosacral junction and was not really tested for corpectomy situations, such as performed here.

Of course, the surgery described in this case report was performed flawlessly by highly experienced surgeons who apparently proceeded with attention to detail and presumably encountered adequate bone stock in their patient. Case reports like these may, however, invite undue imitation and lead to unfavorable outcomes in the hands of less experienced and detail-oriented surgeons.

The EBSJ editorial team congratulates Crabtree and colleagues for their fine work and excellent result. However, based on current literature we side with Schuster in recommending the more predictable anterior debridement and posterior reconstruction approach for this challenging issue. 